\title{
CORRESPONDENCE
}

\section{Does the newly observed inflammatory syndrome in children demonstrate a link between uncontrolled neutrophil extracellular traps formation and COVID-19?}

Pediatric Research (2021) 89:716-717; https://doi.org/10.1038/s41390020-0996-1

The British National Health Service (NHS) issued an alert notifying doctors of a small but growing number of cases of "multisystem inflammatory" disease in children that required intensive care. Given that only about a dozen such cases have been reported in the UK, this inflammatory syndrome appears so far to be rare. Symptoms include fevers, severe abdominal pain and skin rashes, along with markers of severe inflammation in the blood. Some of these children have also needed treatment for heart inflammation. Apart from one child in England who has died, rapid and appropriate treatment has rapidly improved the children's clinical condition. Physicians in the US, Spain, Italy and France have reported similar cases.

This is a very rare disease, but given the current pandemic conditions, it is entirely plausible that at least some of these cases are due to SARS-CoV2. Some of these children have tested positive for COVID-19, while others have not. These cases show symptoms similar to those found in a rare condition known as the Kawasaki disease (KD). The KD is a childhood illness that causes inflammation in blood vessel walls, and that in serious cases can cause heart damage.

As recently reviewed by Lo, ${ }^{1} \mathrm{KD}$ is a common vasculitis in childhood, typically affecting children under the age of 5. So far, no causative infectious agent has been identified for it, despite many years of intense scrutiny. The strongest hypotheses to date as to its cause are viral infection or toxin-mediated reaction to autoinflammatory disease. KD occurs typically as an acute, selflimited, hyperinflammatory state, characterized by distinctive clinical features. These include vasculopathy that principally targets the coronary vasculature, thus potentially increasing risk for early atherosclerosis and ischemic heart disease. Rowley et al. $^{2}$ have identified in KD the upregulation of multiple immune pathways, including intracellular cell signaling, T-cell activation, B-cell development, and type I IFN, suggesting the activation of both an innate and adaptative immune response.

$\mathrm{KD}$ pathogenesis also appears to be associated with a certain genetic background, with infectious trigger pathogen-associated molecular patterns (PAMPs) or damage-associated molecular patterns (DAMPs), and with certain environmental factors. ${ }^{1}$ With respect to the adaptative immune response, recent studies have revealed that $\lg A$ response in $K D$ may be an important contributor to vascular damage, in particular to the induction of increased intestinal permeability. ${ }^{1}$ With regards to the innate immune response, KD is characterized by high levels of IL- 1 that is central to the acute inflammatory phase. IL-1 directly activates coronary artery endothelial cells, resulting in the upregulation of cell adhesion molecules and the production of IL- 6 and IL-8. ${ }^{1}$ Activation of the NLRP3 inflammasome (a complex of three subunits that detect PAMPs or DAMPs) mediates the stimulation of IL-1 production. ${ }^{3}$ Besides the FcyRIIA receptor gene, KD pathogenesis also appears to involve the gene encoding for inositol 1,4,5-triphosphate-3-kinase C (ITPKC), an enzyme that negatively regulates the calcium response in immune cells. ${ }^{4}$ Interestingly, the NOD1 ligand was capable of causing vasculitis even in the absence of a functioning adaptive immune system. ${ }^{5}$ A better understanding of the innate immune dysregulation appears vital in delineating KD pathogenesis, given the major role the innate immune response seems to play.

The fact that no unifying infection has been found favors a multiple-agent model in KD. Unlike most infections, however, there are significant differences in ethnic predilection, which suggests a strong genetic influence. ${ }^{1}$ In genome-wide association studies (GWAS), the most significantly and repeatedly associated susceptibility genes of $\mathrm{KD}$ have, to date, been identified as FCGR2A, BLK, CD40, ITPKC and CASP3. ${ }^{1,6}$ Note, SNPs of ITPKC and CASP3 have never been associated in GWAS for diseases other than KD. ${ }^{6}$ Nevertheless, we believe that they may both have a role in neutrophil/NETs (neutrophil extracellular traps) regulation. Both BLK and CD4O have been known as susceptibility genes for multiple autoimmune diseases, for instance rheumatoid arthritis (RA) and systemic lupus erythematosus, which are NETs-associated diseases. CASP3 and CD4OL have been identified as NET regulators. ${ }^{7}$ Onouchi ${ }^{6}$ reported that $\mathrm{KD}$ patients with susceptibility alleles of ITPKC and/or CASP3 were more likely to develop coronary artery lesion. Despite previous efforts on GWAS, as well as certain trends reported among different populations, ethnicity-specific association was not clearly identified. The involvement of rare variants and mutations is suggested in the literature. ${ }^{6}$ This highlight the dependence of KD on multiple factors, as delineated by Lo. ${ }^{1}$ Nonetheless, several observations point towards the dysfunction of the innate immune response, in particular NETs formation and by-products.

We speculated that the dysregulation of NETs formation caused by SARS-CoV2 infection plays a key role in KD.7 NETs are web-like DNA decorated with antimicrobial proteins, which trap and kill microorganisms. ${ }^{8}$ NETs are essential for innate immunity. Despite strict regulation by serum factors, dysregulation of their formation may have serious adverse effects, due to the systemic release of DNA, histones and granular proteins, which act as autoantigens to the host. ${ }^{9,10}$ This can lead to various diseases such as thrombosis, cystic fibrosis, sepsis, transfusion-related acute lung injury, severe obesity, gouty arthritis, pre-eclampsia or kidney diseases; and in the pathogenesis of autoimmune diseases such as lupus, type 1 diabetes, vasculitis or rare conditions affecting small blood vessels, particularly those of the lungs, skin and kidneys. ${ }^{7}$

Yoshida et al. ${ }^{11}$ recently reported in Pediatric Research that spontaneous NET formation was enhanced in neutrophils from patients with acute KD. This observation associates KD with NETs formation, and supports our hypothesis that NETs and NET by-products play a key role in COVID-19 pathogenesis. ${ }^{7}$ We have 
recently demonstrated that the pathologies and biological features of COVID-19 are analogous with those caused by the deleterious effects of uncontrolled NETs formation. NETs and NET by-products may play a key role in COVID-19 pathogenesis. ${ }^{7}$ Both COVID-19 and the disorders associated with dysregulated NETs formation are complex diseases that result in inflammatory diseases and multiorgan damage. More specifically, both are associated with an abnormality of coagulation factors, prothrombotic activity, and with cytoxicity towards endothelial and epithelial cells, leading in particular to systemic vascular permeability. In addition, we found that NETs dysregulation and byproducts are associated with other COVID-19 comorbidities, such as pulmonary diseases, obesity, inflammatory bowel diseases, cardiovascular diseases, cystic fibrosis, Sickle cell disease, diabetes, neuropathy and rheumatoid arthritis. ${ }^{7}$

It is therefore reasonable to speculate that uncontrolled NETs formation is a link between KD and COVID-19. As a consequence, serious consideration should be given to the possibility that this newly observed syndrome occurring in children is a signal of COVID-19. In addition, as regards therapeutic responses to the pandemic, it supports our conviction as to the importance of investigating new strategies that target NETs and NET by-products.

\section{ACKNOWLEDGEMENTS}

The authors thank S. Dejasse, C. Mc Carthy. A.R.T. is supported by INSERM. This work was funded by the "SIRIC Montpellier Cancer Grant INCa_Inserm_DGOS_12553".

\section{ADDITIONAL INFORMATION}

Competing interests: The author declares no competing interests.
Publisher's note Springer Nature remains neutral with regard to jurisdictional claims in published maps and institutional affiliations.

Alain R. Thierry ${ }^{1}$

${ }^{1}$ Research Institute of Cancerology of Montpellier, INSERM U1194, IRCM, ICM, Montpellier University, 34298 Montpellier, France Correspondence: Alain R. Thierry (alain.thierry@inserm.fr)

\section{REFERENCES}

1. Lo, M. S. A framework for understanding Kawasaki disease pathogenesis. Clin. Immunol. 214, 108385 (2020).

2. Rowley, A. H. et al. The transcriptional profile of coronary arteritis in Kawasaki disease. BMC Genomics 16, 1076 (2015).

3. Swanson, K. V., Deng, M. \& Ting, J. P.-Y. The NLRP3 inflammasome: molecular activation and regulation to therapeutics. Nat. Rev. Immunol. 19, 477-489 (2019).

4. Alphonse, M. P. et al. Inositol-triphosphate 3-kinase $C$ mediates inflammasome activation and treatment response in Kawasaki disease. J. Immunol. 197, 3481-3489 (2016).

5. Nishio, $\mathrm{H}$. et al. Nod1 ligands induce site-specific vascular inflammation. Arterioscler. Thromb. Vasc. Biol. 31, 1093-1099 (2011).

6. Onouchi, Y. The genetics of Kawasaki disease. Int. J. Rheum. Dis. 21, 26-30 (2018).

7. Thierry, A. \& Roch B. NETs by-products and extracellular DNA may play a key role in COVID-19 pathogenesis: incidence on patient monitoring and therapy. Med. Pharmacol. https://doi.org/10.20944/preprints202004.0238.v1 (2020).

8. Elkon, K. B. Review: Cell death, nucleic acids, and immunity: inflammation beyond the grave. Arthritis Rheumatol. 70, 805-816 (2018).

9. Cheng, O. Z. \& Palaniyar, N. NET balancing: a problem in inflammatory lung diseases. Front. Immunol. https://doi.org/10.3389/fimmu.2013.00001 (2013).

10. Schönrich, G. \& Raftery, M. J. Neutrophil extracellular traps go viral. Front. Immunol. https://doi.org/10.3389/fimmu.2016.00366 (2016).

11. Yoshida, Y. et al. Enhanced formation of neutrophil extracellular traps in Kawasaki disease. Pediatr. Res. https://doi.org/10.1038/s41390-019-0710-3 (2020). 